\title{
De l'analyse de l'activité des enseignants débutants en milieu difficile à la conception de dispositifs de formation
}

Luc Ria

Ria, L. (2009). De l'analyse de l'activité des enseignants débutants en milieu difficile à la conception de dispositifs de formation. In M. Durand \& L. Filliettaz (Eds.), Travail et Formation des Adultes (pp.217-243). Paris : PUF.

\section{Le risque d'une usure précoce des enseignants du second degré en France}

Trois quarts des professeurs du second degré « font leurs classes » dans des académies peu convoitées (Guibert, Lazuech \& Rimbert, 2008). Celles de Créteil ou de Versailles en accueillent chaque année plus de 3000 et $40 \%$ d'entre eux sont affectés dans des établissements classés dans le plan de relance de l'éducation prioritaire ${ }^{1}$ que leurs aînés s'empressent de quitter. Au bout de sept ans, deux tiers des débutants repartent de ces académies d'accueil. Il en résulte que dans certains établissements, $40 \%$ des enseignants ont moins de deux ans d'ancienneté, soit un taux de rotation des enseignants de $80 \%$ tous les deux ans. Cette gestion quasi immuable du personnel du second degré repose sur la conviction que ces jeunes enseignants sont " plein d'ardeur et d'énergie » (Livret vert, 2008) et qu'il suffit de les accompagner pour qu'ils s'adaptent à leur nouveau métier. Cependant, les conditions de vie parisienne et de travail face à des publics scolaires très hétérogènes sont généralement plus difficiles que celles rencontrées jusque-là dans leur académie d'origine. Une enquête récente ${ }^{2}$ montre que les jeunes enseignants des collèges d'Ile de France sont plus fatigués que la moyenne nationale par leurs conditions d'exercice professionnel: près des trois quarts (71\%) ont consulté un médecin dans les 24 derniers mois pour « fatigue due au travail ». Cette fatigue s'explique en grande partie par le caractère très contraignant de l'exercice de leur métier, notamment en collège.

A cet égard, une étude longitudinale a permis de modéliser la dynamique de l'activité professionnelle en classe et hors classe d'une dizaine de néo-titulaires de plusieurs disciplines d'enseignement exerçant dans un collège ambition réussite (Rouve \& Ria, 2008). Ceux-ci ont évoqué durant l'année scolaire quatre sources principales de perturbation, de déstabilisation de leur activité professionnelle à l'origine d'un surcroît de fatigue et de doutes identitaires : a) l'agitation des élèves nécessitant un surinvestissement pour prendre ou reprendre le contrôle de leurs classes, b) l'implication dans de multiples projets scolaires transdisciplinaires se développant de manière caractéristique dans les établissements ambition réussite et générant un surinvestissement au sein de l'établissement mais aussi la perte des repères à peine construits avec leurs propres classes, c) le rôle ambivalent du collectif d'enseignants très jeunes, parfois vecteur d'intégration professionnelle, parfois vecteur de conflits identitaires et de fuites et d) le désagrément persistant pour les provinciaux d'une installation précaire dans un contexte urbain peu familier. La fatigue est apparue dans les journaux de bord, utilisés

\footnotetext{
${ }^{1}$ Initiée en 1981, la politique des ZEP a pour objet de renforcer l'action éducative dans les zones où les conditions sociales constituent un facteur de risque, voire un obstacle, pour la réussite scolaire des enfants et adolescents qui y vivent. Cette politique correspond à une mutualisation des moyens et une coordination des actions éducatives. Depuis 2005, des critères nationaux ont été retenus pour découper l'éducation prioritaire en trois niveaux (situation sociale des familles, retard scolaire des élèves, évaluation des résultats à l'entrée en collège, nombre d'élèves non francophones).

${ }^{2}$ Sgen/Cfdt (2008) sur les difficiles conditions de travail des enseignants débutants en Ile de France.
} 
pour consigner leur expérience professionnelle quotidienne, dès les premières semaines de cours. Le surmenage, la sensation d'usure ont conduit la majorité des enseignants observés à pondérer en cours d'année scolaire leur implication dans de multiples projets. La diminution de leurs exigences éducatives et leurs attitudes de protection de soi sont apparues comme les signes d'une « usure professionnelle passagère » en fin d'année scolaire.

Parmi l'ensemble des contraintes régulièrement évoquées par les néo-titulaires, la gestion de classe, en particulier la discipline et la mise au travail des élèves, apparaît comme une des principales difficultés professionnelles (Guibert, Lazuech \& Rimbert, 2008). Un tiers d'entre eux estiment que la composante essentielle de leur métier est moins de transmettre des savoirs que de faire de la discipline. C'est dans ce contexte de difficulté croissante du métier (Maroy, 2006) que les néo-titulaires du second degré remplacent massivement leurs aînés. Si certains s'adaptent de manière remarquable à des conditions d'enseignement difficiles, d'autres éprouvent des difficultés récurrentes pendant plusieurs années et font le plus souvent le deuil des exigences scolaires et d'une conception idéalisée de leur métier. Le risque d'une telle entrée professionnelle, sans qu'une familiarisation préalable à l'enseignement en milieu difficile ne soit effectuée ni que de véritables modalités d'accompagnement ne soient développées, est de voir ces jeunes enseignants s'user prématurément et quitter en nombre l'enseignement comme le font déjà $40 \%$ de néo-enseignants anglais ou américains quelques années seulement après leur prise de fonction (Smethem, 2007). Actuellement en France, si presque la moitié des débutants estiment vouloir changer de métier dans les années à venir, une proportion infime (moins de 5\%) quitte de manière précoce l'enseignement du second degré.

\section{Un enjeu pour la formation des enseignants}

\subsection{L'utilité discutée de la référence experte pour former les novices}

Les Instituts Universitaires de la Formation des Maîtres (IUFM) ont pour mission de préparer le plus efficacement possible les futurs enseignants aux conditions d'exercice professionnel en grande partie renouvelées. Ce qui nécessite d'adapter, d'ajuster les modules de formation à l'évolution de la professionnalité enseignante (Gelin, Rayou \& Ria, 2007). Mais cette mission comprend au moins trois paradoxes qui en limitent l'efficacité. Le premier vient du fait que les stagiaires sont moins dans de véritables processus d'apprentissage du métier qu'à la recherche de stratégies de masquage voire d'évitement inhérentes aux processus de certification qui pèsent encore sur eux en fin de formation initiale. Le second paradoxe est lié au fait que ces derniers se contentent la plupart du temps de stabiliser, de pérenniser leur activité professionnelle dans des contextes de stage moins durs, dans leurs académies d'origine, que ceux vers lesquels ils se destinent à la rentrée scolaire suivante. La quête de l'action efficace se limite aux situations de classe hic et nunc faute de pouvoir anticiper - concrètement ou virtuellement - celles plus difficiles à venir. Cette méconnaissance de l'horizon professionnel des académies d'accueil génère de très nombreuses inquiétudes chez les néo-titulaires du second degré et un véritable choc pour beaucoup d'entre eux lors des premières semaines d'enseignement.

Le troisième paradoxe est lié à la mobilisation quasi systématique en formation de l'activité experte comme référence pour analyser celle des novices; ce qui génère une efficacité toute relative. D'une part, les conseils prodigués par des enseignants chevronnés s'ils mobilisent leur propre vécu semblent peu impacter l'activité des novices qui s'estiment souvent être très éloignés des préoccupations professionnelles de leurs aînés. D'autre part, l'utilisation en formation d'extraits vidéo de l'activité expérimentée est moins prisée par les débutants que la mobilisation d'extraits vidéo de pairs au travers desquels ils se reconnaissent et se projettent bien davantage. Ces constats s'expliquent en partie par le fait que les novices et les plus chevronnés ne perçoivent pas les situations d'enseignement de la même façon : les 
premiers tentent de s'adapter en permanence à des situations nouvelles imprévisibles en masquant leurs surprises et leurs émotions ; les seconds agissent dans un environnement familier en repérant les indices typiques de situations déjà vécues et en mobilisant à tout instant des connaissances construites antérieurement. L'activité débutante analysée et interprétée à l'aune de celle des plus expérimentés conduit davantage à une caractérisation en creux de ce que le débutant n'est pas qu'à une réelle investigation de ce qu'il est, ce qu'il ressent et interprète en situation professionnelle. Ce qui limite les possibilités de l'inscrire dans un projet de transformation réaliste à court terme.

\subsection{Des dispositifs de formation indexés aux mondes typiques des débutants et aux pratiques sociales-cibles}

Pour tenter de dépasser ces difficultés, notre conception de la formation s'appuie sur des corpus de recherche s'efforçant de décrire "les mondes typiques » des débutants avec leurs émotions typiques (Ria, Sève, Theureau, Saury \& Durand, 2003), leurs préoccupations et dilemmes typiques (Ria, Saury, Sève \& Durand, 2001), leurs expériences typiques (Ria, Sève, Durand \& Bertone, 2004) recoupant seulement partiellement les mondes des enseignants chevronnés.. Ne pas prendre en compte ces différences de signification conduit à définir des objectifs de formation pour les entrants dans le métier inatteignables à ce momentlà de leur trajectoire professionnelle et non pertinents par rapport à leurs mondes propres (Leblanc, Ria, Dieumegard, Serres \& Durand, 2008).

Notre cadre d'analyse de l'activité des débutants s'inscrit dans le paradigme de «l'action ou la cognition située », s'attachant à décrire du point de vue de l'ergonomie cognitive, l'organisation intrinsèque du «cours d'action » (Theureau, 2004, 2006), c'est-àdire la dynamique de construction de signification des acteurs dans le cours de leur activité. Quatre présupposés essentiels le caractérisent. Premièrement, l'activité est indissociable de la situation dans laquelle elle prend forme (Varela, 1989) et l'acteur participe à la construction de cette situation. L'activité se caractérise par une succession d'états transitoires d'équilibre et de déséquilibre. Son étude revient à analyser l'histoire des couplages acteur/situation en repérant les éléments stabilisateurs ou déstabilisateurs. Deuxièmement, l'activité s'accompagne d'une conscience préréflexive (Theureau, 2006), dont l'acteur peut rendre compte, au moins en partie moyennant des conditions favorables de collaboration. Cette conscience préréflexive n'est pas ajoutée à, mais constitutive de son activité. L'accès contrôlé et raisonné à l'expérience permet de mettre à jour les ressources cachées de l'action.

Troisièmement, l'activité donne lieu à la construction de connaissances sur la base d'inférences validant/invalidant des connaissances antérieures et en construisant de nouvelles en relation avec l'efficacité pragmatique des actions réalisées dans la situation présente. Ces inférences s'appuient sur des processus de typicalisation (Rosch, 1978) par lesquels les acteurs catégorisent des situations, événements ou actions sur la base de jugements de typicalité. Ils s'appuient sur l'identification d'un faisceau de ressemblances ou de différences entre expériences successives pour juger de cette similarité. Quatrièmement, l'activité exprime à la fois une singularité et une appartenance à une communauté (Lave \& Wenger, 1991). Bien que ressentie comme singulière et subjective, une activité se déploie dans la plupart des cas sur la base d'un héritage culturel qu'elle se ré-approprie et dont elle porte l'empreinte par le biais de processus de sémiotisation de l'expérience (Bronckart, 2002).

A partir de cette conception enactive de l'activité (Durand, 2008; Leblanc et al., 2008), notre approche de la formation reprend les visées de formation indexées à l'analyse de l'activité réelle de la didactique professionnelle (Pastré, 1995, 2008). Elle s'efforce d'articuler de manière consubstantielle des visées épistémiques et transformatives (Ria, Leblanc, Serres $\&$ Durand, 2006) en proposant comme contenus de formation des pratiques sociales-cibles mais aussi des modélisations par la recherche des modalités d'adaptation des enseignants à 
ces pratiques sociales-cibles. L'espace de formation favorise les aller et retour entre les expériences professionnelles des enseignants en formation et les extraits vidéo de l'activité des enseignants en milieu difficile. Ces artefacts de formation ont pour objectif de venir perturber leur activité régulière, d'infléchir leur cours d'action. La simulation de scénarios fictifs mais plausibles, virtuels mais potentiellement réalisables, a pour ambition de participer à la transformation anticipée des dispositions à agir de ces enseignants en formation par des processus de typicalisation mais aussi en faisant appel à leur imagination et créativité. Cette démarche de formation amène à reconsidérer les rôles du formateur et des formés (Durand, Ria \& Veyrunes, à paraître) et à envisager le processus de formation plus activement sur une temporalité longue. Cependant, bien qu'accompagné dans les centres de formation, le développement de l'activité des enseignants débutants donne lieu à des transformations singulières et asynchrones qui échappent pour partie aux formateurs (Serres, Ria \& Adé, 2004). Il ne nous semble ni possible ni utile de chercher à les contrôler, mais il est par contre primordial de concevoir des formations offrant à chaque formé des opportunités d'autonomie et de développement en lien avec ses objectifs, intérêts et curiosités, et avec les objectifs des instituts de formation.

\section{Un observatoire de l'activité des novices en milieu difficile}

C'est pour tenter de rapprocher les dispositifs de formation initiale de la réalité d'un métier en pleine transformation, qu'un observatoire de recherche ${ }^{4}$ a été conçu sur le travail de jeunes enseignants dans les établissements scolaires de la périphérie parisienne classés dans le plan de relance de l'éducation prioritaire (Ria \& Rayou, 2008). Le choix de ce terrain d'étude est lié à deux raisons principales. La première tient au fait que les néo-titulaires rencontrent pour la première fois des publics scolaires se caractérisant autant par leur très grande hétérogénéité scolaire que par leur résistance à l'égard du travail scolaire. Notre observatoire s'attache à comprendre comment ils s'adaptent à ces nouveaux publics. La seconde est liée au fait que les établissements inscrits dans le plan de relance de l'éducation prioritaire (2006) bénéficient de moyens humains et financiers supplémentaires. Par exemple, dans les établissements classés Ambition Réussite, les néo-titulaires bénéficient d'un accompagnement par des professeurs plus expérimentés nommés professeurs référents. Ces conditions matérielles et humaines majorées peuvent favoriser l'émergence de pratiques innovantes en classe (par exemple, des interventions à plusieurs enseignants devant les élèves), de nouvelles dynamiques de travail entre les enseignants sur des projets éducatifs (par exemple, la mise en place pour toutes les classes de sixième de parcours individualisés pour l'accès aux compétences du socle commun en français ou en mathématiques). Ces établissements peuvent devenir alors de véritables laboratoires d'innovations pédagogiques portées par des enseignants très investis dans leur mission d'accompagnement des élèves les plus défavorisés du système éducatif français.

L'ensemble de ces transformations de l'activité professionnelle en milieu difficile constitue à notre sens un bon indicateur des évolutions probables du système éducatif français à plus long terme, du changement de la professionnalité enseignante, notamment du fait des caractéristiques de ces enseignants qui ne sont pas seulement jeunes mais " nouveaux » par rapport à leurs prédécesseurs puisqu'ils n'ont eux-mêmes connu, comme élèves, qu'un enseignement massifié (Rayou \& van Zanten, 2004). Ainsi, la documentation de leur travail réel a pour but de participer au processus de re-normalisation des prescriptions énoncées en formation, en faisant des changements plus ou moins perceptibles de l'activité professionnelle des objets d'étude et d'interrogation en phase avec les attentes et les préoccupations des futurs enseignants.

\footnotetext{
${ }^{4}$ En collaboration avec Patrick Rayou, sociologue de l'éducation (Paris 8) et l'IUFM de Créteil depuis 2005, puis le Centre de Ressources Alain Savary de l'INRP depuis 2007.
} 


\subsection{Modélisation hiérarchique des dispositions à agir en début de cours}

Presque la moitié des enseignants affirment après 5 années d'expérience éprouver encore des difficultés importantes pour mettre les élèves au travail en début de cours (Guibert et al., 2008). Il s'agit pour eux d'un point noir récurrent tout au long de l'année scolaire. Le suivi d'une vingtaine d'enseignants débutants tout au long de l'année scolaire a permis d'identifier trois dispositions à agir typiques hiérarchisées selon des critères d'efficacité lors de l'entrée en classe des élèves et de leur mise au travail. Ces dispositions à agir (Lahire, 1998) sont comprises comme l'ensemble des composantes perceptives, interprétatives, cognitives, émotionnelles, intentionnelles et actionnelles mobilisé dans une classe de situations. Elles constituent des potentialités régulières et reproductibles dans le processus d'adaptation des acteurs à leur environnement qu'ils considèrent selon leurs expériences comme typique ou non. L'action produite correspond à l'actualisation d'une disposition à agir structurée, significative et réglée selon les circonstances de son émergence. Dans cette perspective, la notion de disposition à agir condense et articule déjà-là et émergence, potentialité et actualité, conservation et invention (Durand, 2008).

Chaque disposition à agir relative à l'entrée et la mise au travail des élèves se distingue de la précédente par la modification de ses composantes mais aussi par les effets qu'elle génère sur la situation scolaire appréhendée tant du point de vue de l'ambiance générale de la classe que de celui des comportements individuels des élèves. Chacune d'entre elle correspond à des types d'activités différents - gradués les uns par rapport aux autres dont les descriptions permettent de comprendre la dynamique de transformation au sein d'une même disposition à agir. La dynamique de transformation entre deux dispositions à agir exprime plus largement le « changement de monde » des enseignants qui mobilisent lors de ce passage d'autres repères pour percevoir et interpréter la classe, d'autres registres d'action.

\section{Disposition à agir 1 : une mise à l'écart des savoirs scolaires}

L'activité typique 1 de la première disposition à agir caractérise les enseignants en grande difficulté en début de cours avec leurs élèves. Lors de ces situations scolaires non contrôlées, ils ressentent des émotions intenses et s'estiment démunis de solutions efficaces pour amorcer la mise au travail de leurs élèves: "Je ne sais pas comment faire pour démarrer mon cours... Je ne me préoccupe pas du tout du contenu de la leçon... ». Leurs élèves continuent d'adopter en classe les mêmes comportements que ceux observés quelques minutes auparavant dans les couloirs de l'établissement (bousculades, railleries et interpellations à haute voix). Les enseignants attendent en vain les conditions favorables à la mise au travail des élèves. Les élèves s'esquivent, résistent et poursuivent leurs activités clandestines de manière parfois provocante. Les enseignants expriment leur désarroi et renoncent par rapport à leurs préparations de cours : "Je suis inquiète, les élèves n'adhèrent pas à l'exercice, je change, inutile d'insister... ». Leurs interventions verbales véhémentes restent sans effet.

L'activité typique 2 traduit une première transformation notable dans la façon d'accueillir les élèves. Les enseignants, selon des échelles temporelles propres à chacun (jours, semaines, mois), ont construit avec les élèves leaders des «pactes de non agression réciproques ": "J'essaye de ne pas rentrer en conflit avec ces élèves. Le dialogue est nécessaire pour ne pas les braquer...». Des règles de vie de groupe sont respectées, l'ambiance de classe y est plus sereine, mais le scénario de début de cours encore incertain. Les enseignants reconnaissent leur incapacité à provoquer de leur propre chef la mise au travail : "Je sais qu'ils vont se mettre à travailler... J'attends sans savoir combien de temps cela va durer ... ». Ce qui peut, dans certains cas extrêmes, durer toute l'heure : les élèves, 
experts de ces situations d'attente, de ces transitions aux contours mal définis, multiplient les activités parallèles pour retarder la mise au travail.

Cette première disposition à agir résulte de plusieurs couplages typiques relatifs aux premières expériences des débutants en milieu difficile. Qu'il s'agisse du chahut récurrent d'une majorité d'élèves ou des perturbations isolées d'élèves leaders de la classe, ces débuts de cours se caractérisent par des négociations incessantes, des attentes interminables. Si les enseignants construisent des connaissances sur les élèves perturbateurs et la façon d'instaurer des formes de modus vivendi en classe, ils ont tendance à mettre leur discipline d'enseignement à l'écart, entre parenthèses, le temps d'établir l'ordre en classe. Ce qui repose sur la conviction qu'il est nécessaire d'attendre l'installation d'un climat de classe suffisamment propice à la délivrance des consignes pour garantir l'efficacité du travail scolaire. Les enseignants tentent alors en début de cours de réduire le chahut [activité typique 1] ou de contrôler par le dialogue les élèves les plus difficiles [activité typique 2] sans trouver de solution pour les mettre au travail plus rapidement.

\section{Disposition à agir 2 : des savoirs scolaires détournés à des fins d'économie personnelle}

La deuxième disposition à agir s'échafaude de manière empirique, par essais et erreurs, en réaction aux premières expériences de classe douloureuses. Les enseignants coupent court à toute négociation et déploient des stratégies parfois brutales d'entrée en classe. L'activité typique 3 s'inscrit dans un registre compensatoire : elle se traduit par l'enrôlement autoritaire des élèves à peine entrés dans la classe dans des tâches écrites souvent de bas niveau d'exigence scolaire (recopiage ou textes à trous) : " Au bout de deux mois [mois de novembre], après avoir essayé plein de trucs, j'ai réussi à reprendre la main en les faisant écrire systématiquement en début de cours... ». Les interventions en classe y sont parfois cinglantes, traduisant chez certains enseignants la peur d'être (encore) chahutés : "En fait, $j$ 'ai toujours peur que ça parte en vrille [chahut], toujours... ». L'usage de l'écrit s'effectue par défaut. Les débutants n'ont pas forcément pris conscience de manière explicite que l'ordre en classe peut être la conséquence du travail des élèves et non l'inverse, mais ils adoptent intuitivement « ce qui marche »: "Ça les calme, je passe dans les rangs, je contrôle le travail... ».

Parfois, la prise de conscience est collective comme pour ces deux néo-titulaires d'anglais lors d'un dispositif d'observation entre pairs organisé dans le cadre de leur accompagnement à l'entrée dans le métier dans l'Académie de Créteil. L'une d'entre elle constate la difficulté récurrente de débuter le cours par un exercice collectif à l'oral comme le préconisent les orientations officielles de cette discipline : "Et là, je me rends compte que l'institution a ses limites quand même... Ça crée un climat d'excitation [l'oral en début de cours]... J'aurais du faire un truc écrit en premier... En voyant... Maintenant je ne le ferai plus jamais [en tapant du poing sur la table]... Je sens que la prochaine fois, si les élèves arrivent et qu'ils sont surexcités comme ça, je vais me préparer une batterie de petites activités écrites, faites rapidement en 5 minutes que je sortirais à chaque début d'heure si jamais je sens que ça va être le bazar. Je ferai un truc à l'écrit en premier et après l'oral, un truc en rapport avec ce que l'on fait à l'oral... ».

L'activité typique 4 repose toujours sur la conviction que le travail écrit individuel avec des consignes minimales peut contribuer de manière efficace à la diminution du bruit et à la prise en main progressive de la classe dans son ensemble. Mais, cette fois, son emploi est plus structuré, les exercices scolaires sont plus sophistiqués que le simple recopiage d'un cours au tableau ou le remplissage d'un texte à trous. Les configurations d'entrée en classe s'organisent sur plusieurs variables : répartition des élèves leaders, choix d'exercices attractifs et accessibles, modalités d'évaluation du travail écrit, délégation de rôles spécifiques pour les élèves, etc. Ces configurations ritualisées neutralisent l'agitation réelle ou potentielle tout en 
offrant des possibilités d'action aux enseignants dans d'autres registres (supervision à distance, anticipation de la suite de la leçon, etc.). Les enseignants n'hésitent pas à reconnaître l'intérêt personnel qu'ils portent à ces modalités d'organisation des débuts de cours : "Là, c'est calme, c'est ce que je recherche, moi ça me permet de me reposer ou de penser à plein de choses... En fait, je fais semblant de les surveiller en me déplaçant, le cahier c'est un peu un prétexte [en faisant semblant de les évaluer]».

Cette seconde disposition à agir se caractérise par l'enrôlement des élèves le plus tôt possible par le travail individuel pour favoriser in fine les conditions nécessaires au travail de toute la classe. Ces modalités sont rarement préconisées en formation initiale. Les exercices scolaires y sont en quelque sorte détournés de leur fonction première, instrumentalisés à des fins de protection de soi : la recherche pragmatique d'une stabilité de l'activité en classe (même ponctuelle), d'un plus grand confort d'enseignement est première, la pertinence didactique seconde. Ces configurations de classe permettent une mise au travail bien plus rapide sur des tâches qui constituent des «sas d'accueil» routiniers pour les élèves et qui offrent aux enseignants des marges de manœuvre pour re-stabiliser leur propre activité et anticiper la suite de la leçon.

\section{Disposition à agir 3 : des savoirs scolaires en permanence légitimés}

La troisième disposition à agir en début de cours se caractérise par l'abandon de ces étayages compensatoires pour mettre les élèves au travail. Si certains ne parviennent pas à abandonner totalement l'écrit pour mettre les élèves au travail, ils en font un usage plus circonstancié : "Je ne me sépare pas totalement de l'écrit en début d'heure, mais ce n'est plus systématique. Je ne l'utilise plus seulement pour avoir la paix. Cela me permet d'adapter le déroulement du cours aux moments de la journée ou de la semaine, en fonction du profil des classes ». Les enseignants s'estiment davantage en mesure de prendre la classe sans mobiliser ces configurations qu'ils finissent par trouver trop répétitives ou sclérosantes. Néanmoins, d'autres formes de ritualisation apparaissent [activité typique 5] : les enseignants vont à la rencontre des élèves le plus tôt possible dans les couloirs, instaurent un sas d'accueil individuel devant la porte pour signifier les yeux dans les yeux, par des prises de paroles personnalisées, le changement de territoire et les nouvelles règles qui en découlent. En multipliant les déplacements autour des élèves agités et les remarques individuelles parfois mesurées, parfois théâtralisées, ils parviennent à canaliser l'agitation collective et à percevoir le point de bascule favorable à l'amorce des premières questions sur le travail scolaire en cours : "Il faut que je capte ce début de silence pour prendre la main... Il faut qu'avec une question de maths, toutes les conversations tournent enfin autour des maths..." ". L'enrôlement des élèves dépend alors de leur capacité à susciter l'intérêt, la curiosité, les confrontations interindividuelles. Ils estiment "être dans l'arène », s'épuisant à contenir l'ardeur des réponses bruyantes et souvent décalées par rapport à leurs attentes. L'adhésion des élèves provient de leur très forte implication, d'un rapport presque passionnel avec la matière qu'ils enseignent. Ils ne cessent de légitimer leur propre activité en arguant des bénéfices tirés d'un travail assidu dans leur matière d'enseignement.

Ces trois dispositions à agir en début de cours ne constituent pas des patterns professionnels stables et exhaustifs quelles que soient les conditions d'enseignement: des variations peuvent être observées chez un enseignant au cours d'une même journée avec des classes différentes ou selon les périodes scolaires plus ou moins favorables au travail scolaire. Leur modélisation hiérarchique n'exclut pas non plus des régressions chez un enseignant expérimenté découvrant des publics plus difficiles. Néanmoins, ces dispositions récurrentes à percevoir, interpréter et agir en classe confèrent une relative stabilité à l'activité professionnelle (de plusieurs semaines à plusieurs années), même si son efficacité n'est pas toujours probante du point de vue des acteurs. Les mêmes indices perceptifs, les mêmes 
raisonnements sont mobilisés à la racine d'actions récurrentes dans le même type de situations professionnelles. La transformation de ces dispositions à agir s'effectue le plus souvent à la suite d'un événement saillant ou d'une série d'événements en classe poussant au changement, c'est-à-dire réfrénant la propension au conservatisme des savoir faire routiniers pour agir autrement et retrouver un niveau de stabilité et de satisfaction dans l'exercice professionnel. Si le huis clos de la classe ne favorise que très rarement l'acculturation à d'autres modes d'action collectivement valorisés, l'usage en formation de matériaux sur l'activité d'autres enseignants novices ou d'enseignants plus expérimentés peut faciliter cette prise de conscience de manières d'agir plus efficaces.

\subsection{La référence à l'activité d'enseignants plus expérimentés en milieu difficile ?}

$\mathrm{Si}$ des réserves ont été émises précédemment quant au recours systématique en formation à l'activité d'enseignants chevronnés pour analyser l'activité novice, l'étude de l'activité d'enseignants expérimentés [activité typique 6] dans des conditions analogues d'exercice prend ici tout son sens car elle permet de déceler les marges de progrès potentiels des enseignants débutants les plus efficaces [disposition à agir 3 ; activité typique 5] dans la façon d'intervenir auprès de publics scolaires difficiles. En effet, certains de ces enseignants plus expérimentées ont développé au fil des années des dispositions à agir très efficaces dont l'analyse peut enrichir l'horizon expérientiel des enseignants novices en formation. Ces enseignants expérimentés, en proportion très réduite dans ces établissements, n'ont plus besoin d'instrumentaliser les exercices scolaires pour tenir la classe en début de cours ni d'entretenir des formes excessives d'engagement comme le font les débutants. Deux caractéristiques principales fondent leur efficacité en début de cours.

Premièrement, et de façon surprenante tellement ils font preuve de maîtrise lors les situations scolaires observées, ils appréhendent toujours les débuts de cours dans la mesure où selon eux «tout se joue dans les premières minutes ». Leur appréhension provient de la crainte de ne pas être à la hauteur des exigences qu'ils donnent à leurs élèves à chaque heure. Ces enseignants parviennent à créer des ambiances de classe extrêmement calmes seulement par des regards prolongés pendant de longues secondes, des expressions théâtralisées avec des prises de parole minimales. Ces formes de communication non verbale et de présence physique sont autant de modalités d'existence en classe ayant plus d'effet sur les élèves que les interventions fleuves et virulentes des enseignants moins expérimentés. L'extrait suivant illustre la façon dont une enseignante de français, ayant sept années d'expérience dans un collège Ambition Réussite de la région parisienne, perçoit l'ambiance d'une classe de sixième très agitée et joue sur ses déplacements et ses attitudes avec un regard insistant pour " construire le silence » et favoriser la prise de conscience collective d'un " espace/temps » nécessaire au début de cours : "L'instauration de cette nappe [de silence], cela fait du bien à tout le monde... J'en ai besoin parce que j'ai tellement la tête comme ça, ils m'ont saoulée... $C$ 'est quelque chose qui les unit... Enfin qui nous unit... A un moment donné on est dans un même espace/temps. En fait, c'est tellement agité, c'est tellement l'ébullition dans les couloirs qu'on a besoin tous de cette nappe-là [de silence] pour travailler ensemble. Parce que le collectif est issu de cette nappe-là où l'on fait tous quelque chose ensemble, où l'on vit quelque chose sur un texte qui peut être assez fort et qui nous unit... Parce que l'idée c'est qu'on travaille tous ensemble dans une même direction... ».

Deuxièmement, ces enseignants plus expérimentés jouent avec subtilité sur les rapports de force entre les individus et le groupe-classe en tentant de provoquer, parfois de manière très calme, parfois de manière cinglante, des sentiments de culpabilité chez l'élève réprimandé pour qu'il comprenne qu'il perturbe, pénalise, non pas l'enseignant, mais le travail des autres élèves. C'est le cas pour une enseignante de musique, ayant neuf années d'expérience dans un collège Ambition Réussite de la région parisienne, qui, lors d'une 
" colère de prof ", s'appuie dans son intervention sur le collectif des élèves pour tenter de mettre en défaut un élève perturbateur : "Quand je vois que le regard ne suffit pas, alors là je m'énerve [tonalité très forte mais contrôlée] ... Là, il est important que quand je m'énerve, je continue à le regarder [l'élève perturbateur] et en même temps, sans regarder Camilla je la montre [de la main], sans regarder Emilien je le montre, sans regarder Fatima, je la montre... Je prouve là que j'ai la conscience de ces élèves-là... Que même si je ne les regarde pas, je sais qu'ils existent, je sais qu'ils sont là, donc je les remets en valeur et Eddy [l'élève perturbateur], je le remets en question, enfin j'essaye... ».

\section{Des dispositions à agir typiques selon des temporalités singulières}

L'étude des activités novices puis de celles des plus expérimentés met en évidence plusieurs formes d'adaptation aux contextes d'exercice professionnel difficiles : des émotions paralysantes lors des premiers face à face à leur théâtralisation pour modifier l'ambiance de classe, des négociations stériles aux interventions par réaction plus répressives, de l'usage d'exercices scolaires instrumentalisés à des fins de protection personnelle à la quête d'une légitimité plus directement liée aux enjeux de savoirs, de la focalisation sur quelques élèves à l'attention portée au collectif au travail. Autant de changements de repères professionnels, autant de prises de conscience le plus souvent solitaires pour aborder ces débuts de cours problématiques. Notre modélisation graduelle des dispositions à agir tente de conserver autant que possible le caractère dynamique, complexe et pluridimensionnel de l'adaptation des enseignants tout en la réduisant afin de rendre intelligibles les principales bifurcations ou transformations qui s'opèrent au fur et à mesure de l'expérience acquise.

Il en ressort que les enjeux mêmes de l'activité professionnelle des enseignants débutants dépassent ceux liés à la seule transmission des savoirs : tout se passe comme si l'activité professionnelle se construisait selon des phases alternativement centrées sur l'économie de soi et sur la recherche d'une plus grande pertinence concernant les savoirs scolaires proposés aux élèves. Les enseignants ne parvenant pas à prendre le contrôle des élèves et à les mettre au travail vivent particulièrement mal ces situations d'exposition d'euxmêmes pendant lesquelles leur légitimité et leur identité sont mises à l'épreuve voire en danger [disposition à agir 1]. L'activité écrite devient l'un des moyens les plus efficaces pour sauver la face en adoptant une position de repli, de protection personnelle quitte à sacrifier un temps la pertinence de ce qui y est enseigné [disposition à agir 2]. Leurs configurations de classe de plus en plus structurées, vécues comme les « ilots » d'une stabilité professionnelle retrouvée, leur procurent le recul et la disponibilité nécessaires pour réinterroger la pertinence de ce qu'ils enseignent. Mais dans cette quête d'objectifs scolaires plus ambitieux, ils reperdent souvent une partie du confort gagné à grande peine [disposition à agir 3].

La question de l'efficacité du travail des enseignants prend alors un double sens : celui lié aux apprentissages de leurs élèves mais aussi et surtout celui lié à leur propre capacité à s'économiser, à inscrire leur investissement dans la durée d'au moins une année scolaire. Si les débutants dépensent une énergie considérable dans l'énonciation de remarques individuelles sans fin ou au mieux avec une portée limitée à quelques élèves, les enseignants plus chevronnés parviennent à interpeller l'ensemble des élèves en mobilisant des principes éthiques dans leurs prises de parole (respect d'autrui, exigences réciproques, équité des sanctions, justice entre élèves, etc.), qui ont des effets indéniables sur la dynamique collective de la classe. Cette éthique en actes produit bien plus d'effets chez les élèves que la manifestation de comportements d'agression ou de protection de soi observées chez des enseignants davantage en difficulté. Plus généralement, ces enseignants parviennent à rendre compatibles une relative économie d'eux-mêmes et des exigences scolaires d'un niveau que beaucoup considèreraient inatteignable avec ce genre de public très hétérogène. 
Si l'identification de ces dispositions à agir permet de repérer des formes développementales typiques chez les enseignants débutants, le passage d'une disposition à l'autre ne s'opère ni de manière totalement spontanée, ni de manière totalement linéaire : il s'effectue plus ou moins explicitement au fil des expériences, des difficultés selon les contextes scolaires et selon des temporalités propres à chaque enseignant. Par exemple, une enseignante d'éducation physique, après avoir été chahutée en début d'année scolaire, a mis moins de deux mois pour construire des configurations d'entrée en classe très efficaces pour contrôler ses classes par une mise au travail des élèves en petits groupes. Une enseignante de mathématique à peine six mois pour imposer aux élèves le travail à l'écrit dès l'entrée en classe et autant de temps pour abandonner cette forme de travail trop infantilisante selon elle. Ces différences d'adaptation (parfois de stagnation) sont liées à leur capacité plus ou moins tenace à endurer des situations scolaires non conformes à leurs attentes, à leur tendance plus ou moins spontanée à conserver des procédures d'intervention peu satisfaisantes de leur point de vue ou au contraire à leur propension à tester en classe de nouvelles formes d'intervention, nécessitant souvent de prendre des risques.

\section{Des mondes successifs de viabilité personnelle}

Les enseignants novices ne s'adaptent pas mécaniquement à un environnement scolaire stable comme le souhaiterait l'institution qui les emploie : ils transforment leur propre activité tout en transformant en retour l'environnement professionnel dans lequel ils agissent. Les deux se modifient sous l'effet des couplages successifs mais selon des vitesses différentes. Si l'environnement scolaire change de manière spectaculaire, notamment depuis l'entrée massive de populations scolaires très hétérogènes, il change moins vite que l'activité des débutants se transforme elle-même pour qu'ils puissent survivre ou tout au moins " résister » au quotidien dans ces établissements difficiles. Ils fabriquent alors des mondes viables dont les normes successives se transforment selon des critères subjectifs provisoires, produits de la dynamique sans cesse renouvelée de leurs couplages aux situations scolaires.

Notre observatoire de l'entrée dans le métier en milieu difficile montre comment les mondes de viabilité des enseignants se transforment de manière typique. Après la mise en danger de leur identité professionnelle lors des premières expériences de classe, ceux-ci tentent de rependre en main les situations scolaires par eux-mêmes, puisqu'ils ne peuvent compter sur personne d'autre, et pour eux-mêmes, pour se protéger et retrouver une certaine légitimité. Ils privilégient alors les actions efficaces, de leur propre point de vue, quelles qu'elles soient, en rejetant (provisoirement) les critères institutionnels qui leur procurent de l'inconfort, qui exposent leur identité. Ils choisissent les exercices scolaires selon leur capacité à occuper les élèves, selon le bruit qu'ils génèrent, le confort qu'ils procurent. Ils recherchent des espaces de travail stables et prévisibles et rejettent ceux qui peuvent potentiellement les surexposer. Leurs mondes de viabilité intègrent ainsi des critères personnels significatifs : confort/inconfort, seuil acceptable de décibels, degré acceptable d'émotion devant les élèves, degré de mise en péril de leur statut et de leur identité, etc. D'autres critères plus formels ne sont pas forcément des éléments discriminants de leur activité : critères d'apprentissage des élèves, degré de pertinence et de maîtrise des tâches scolaires proposées aux élèves. Ce qui fait que pour que leur monde soit viable, certains enseignants font sortir de leur couplage aux situations scolaires les critères de viabilité formels proposés par l'institution et intégrés en formation initiale. Les indicateurs subjectifs de plaisir/déplaisir au travail constituent des formes de gardes fous contre l'usure professionnelle précoce. Sont-ils pour autant des mécanismes de protection suffisants pour exercer ce métier dans la durée dans ces milieux difficiles?

Ces mondes de viabilité demeurent précaires et donc souvent éphémères. Ils reposent sur des convictions tacites dont la pertinence est mise à l'épreuve. Lors des débuts de cours, 
une bonne partie des enseignants, ayant effectué leur formation initiale dans des contextes scolaires a priori plus faciles, ont tendance à attendre que les conditions soient optimales pour mettre les élèves au travail. Les indices prélevés dans la classe sont alors relatifs au silence des élèves, à la présence du matériel scolaire, au rangement des vêtements et des sacs scolaires, et donc à tous les éléments qui entravent cette mise en ordre de la classe. L'attente dans des classes difficiles peut être sans fin ; de nombreux enseignants s'y épuisent. Ceux qui réussissent à débuter les cours plus rapidement dans des conditions similaires ne font pas forcément preuve de davantage d'autorité contrairement à une idée reçue. Par contre, ils ont construit des mondes de signification différents, reposant sur d'autres convictions, d'autres repères. S'ils ressentent de manière syncrétique des ambiances de classe, des atmosphères plus ou moins favorables, ils s'attachent moins aux perturbations des élèves qu'aux opportunités, qu'aux offres de la situation scolaire pour amorcer le travail individuel, pour lancer la première question de cours, pour détourner ainsi les conversations personnelles au profit d'une question collective en relation avec le cours précédent. Ils n'appréhendent plus les débuts de cours seulement en fonction des entraves et obstacles réels mais selon leur potentiel d'exploitation qu'ils ont appris à déceler, convaincus que la situation idéale de délivrance des consignes n'existe pas dans ce genre de classes. L'ordre en classe n'est plus attendu comme préalable mais comme résultante de leur effort d'amorçage du cours. Ce constat met en lumière les deux propriétés fondamentales de toute connaissance professionnelle (Pastré, 2008) : celle prédicative ou discriminative permettant d'identifier dans le réel des objets, des propriétés, et des relations et celle opérative structurant l'orientation pragmatique de l'activité.

Force est de constater l'asymétrie des registres théoriques (connaissances scientifiques) et pragmatiques (connaissances professionnelles) dans le développement des débutants. Leurs connaissances ne sont pas immédiatement opératives pour mettre les élèves au travail. Ce qui peut générer des blocages et des débuts professionnels douloureux. Des formes d'activité se déploient alors à la marge des préconisations de l'institution. Que faire de ces activités marginales ou clandestines, de ces zones d'ombre dans lesquelles les novices s'engouffrent en s'affranchissant des normes formelles, en s'écartant de la ligne tracée par la formation initiale ? Est-ce qu'une activité stabilisée, réifiée d'une génération d'enseignant à l'autre, constitue la réponse la plus pertinente aux changements de l'environnement du travail enseignant? L'activité la plus «normale » n'est-elle pas celle au contraire qui s'adapte, se transforme au gré des circonstances scolaires mais aussi en fonction des changements des nouveaux agents différents de leurs aînés ? Pour éviter l'émiettement des pratiques, le développement de conduites adaptatives à la marge d'exigences minimales, l'institution et les instituts de formation ne peuvent faire l'économie d'une réflexion pour tenter de renormaliser les prescriptions du métier à la lumière de ces écarts, de ces décalages. Ce qui pose en conséquence la question de la normalité des activités des formateurs eux-mêmes.

\section{De la nécessité de former les formateurs au développement professionnel des novices}

Les contextes d'enseignement difficiles exacerbent le potentiel d'adaptation, l'imagination créatrice des enseignants en début de carrière pour survivre au quotidien. La description scientifique de ces dispositions et activités typiques en formation permet de se détacher des «allants de soi» qui structurent encore souvent les formations, et qui circonscrivent les possibilités d'objectiver des renouvellements parfois à peine perceptibles de l'activité professionnelle. L'activité des novices peut être alors conçue non pas seulement comme un précurseur de l'activité des enseignants expérimentés ou une version dégradée de celle-ci (les néo-titulaires peuvent faire preuve eux-aussi d'efficacité relative, contextuelle), mais comme ayant une organisation et une signification propres et un potentiel de création non négligeable. Ces données de recherche constituent des matériaux pour montrer à la 
communauté scientifique la façon dont les enseignants débutants vivent leur métier au quotidien, mais aussi des matériaux pour la formation initiale des enseignants ou pour la formation des formateurs en IUFM.

A ce titre, la présentation en formation de modèles synthétiques et dynamiques regroupant les constituants critiques de l'activité sociale-cible, vécue typiquement de manière problématique, comme c'est le cas pour les débuts de cours, permet soit l'affinement ou la contestation des modèles usuels préconisés en formation, soit la conception de formations innovantes autour de pratiques professionnelles jamais analysées dans la mesure où elles sont émergentes. Cette analyse scientifique en permet la modélisation qui devient un guide pour la conception. Ainsi, la modélisation des différentes dispositions à agir en début de cours offre des pistes précieuses pour la formation initiale des enseignants, notamment pour leur faire comprendre concrètement comment les options pédagogiques structurant l'activité, spécifiques ou invariantes d'une discipline scolaire à l'autre, les configurations de classes comprises comme des offres potentielles d'actions conjointes, peuvent se révéler des atouts robustes pour tenir la classe mais surtout pour enseigner.

Cette approche de la conception de dispositifs de formation requiert de la part des formateurs une bonne connaissance des étapes du développement de l'activité professionnelle des novices. En effet, l'efficacité des conseils prodigués dépendra de la compatibilité avec leurs dispositions à agir actuelles ou en cours de construction. La référence à l'activité experte peut alors être mobilisée comme une ressource ponctuelle compte tenu de sa proximité, dans un registre de situations scolaires particulier, avec la disposition à agir du novice. Sur ce point, les artefacts de formation doivent pouvoir perturber ses dispositions à agir, sans totalement les déstabiliser, pour les enrichir de nouvelles potentialités dans la façon de percevoir, d'interpréter les situations de classe. Ce qui nécessite de positionner de manière prudente l'activité du débutant, sans la stigmatiser ni la réifier, parmi un ensemble de modélisations de trajectoires typiques, de continuums développementaux caractéristiques des novices, pour repérer les marges de progrès potentiels, les changements ou bifurcations possibles en termes de répertoire d'actions. Pour autant, le danger d'une telle conception serait d'aboutir à une euphémisation des prescriptions adressées aux novices en formation, à un relativisme bienveillant mais peu fécond. Il est nécessaire pour éviter cette dérive de baliser les contours des trajectoires probables de l'activité des débutants en proscrivant des limites déontologiquement ou didactiquement non acceptables sans pour autant prescrire de manière dogmatique la " bonne pratique ». Il s'agira plutôt d'encourager certaines actions en acceptant les marges de manœuvres personnelles, les improvisations ou les décalages.

Mais là, au moins deux principes éthiques essentiels doivent être abordés avant de tenter de conduire avec justesse et pertinence toute action de formation à partir des traces de l'activité réelle des enseignants en milieu sensible. Le premier principe est lié aux modalités et au choix des matériaux de recherche en formation. En effet, s'il est nécessaire de prévenir et préparer les futurs enseignants à un métier sans en masquer les difficultés, il semble tout aussi important de ne pas verser dans des analyses misérabilistes ou des perspectives totalement pessimistes. Il s'agit alors de préférer l'utilisation en formation de l'activité professionnelle efficace ou encore d'insister sur la dynamique de transformation d'une activité problématique pour en comprendre les fondements et le développement potentiel, plutôt que de s'attacher à mettre l'accent sur les situations professionnelles dont l'analyse n'apporte aucune piste féconde pour les dépasser.

Le second principe concerne l'utilisation en formation d'extraits vidéo. L'analyse qui en est faite doit s'enrichir de manière systématique des interprétations que les enseignants confèrent eux-mêmes à leur propre activité (entretiens d'auto-confrontation). Cette précaution peut éviter toute interprétation abusive des seuls comportements. D'autre part, il est nécessaire de ne pas évaluer ou interpréter ex abrupto l'activité d'un enseignant mais de la 
mobiliser : a) comme une forme provisoire d'adaptation dont l'étude des autres activités typiques en amont et en aval de celle-ci donne des clefs pour la comprendre et la transformer, et b) comme un élément de compréhension plus large du développement des débutants ; les analyses tenteront alors de dépsychologiser, de dépersonnaliser l'activité professionnelle pour montrer les aspects les plus génériques d'une communauté enseignante débutante, et non les difficultés inhérentes à des enseignants en particulier.

\section{Bibliographie}

Bronckart, J.-P. (2002). La culture, sémantique du social formatrice de la personne. In F. Rastier, \& S. Bouquet (Eds.), Une introduction aux sciences de la culture (pp.175-201). Paris : PUF.

Durand, M. (2008). Un programme de recherche technologique en formation des adultes. Une approche enactive de l'activité humaine et l'accompagnement de son apprentissage/développement. Education \& Didactique, 2(3), 97-121.

Durand, M., Ria, L., \& Veyrunes, P. (à paraître). Analyse du travail et formation : un programme de recherche empirique et technologique portant sur la signification et l'organisation de l'activité des enseignants. In F. Saussez (Ed.), Analyser l'activité enseignante : des outils méthodologiques et théoriques pour l'intervention et la formation. Québec : Presses de l'Université de Laval.

Gelin, D., Rayou, P., \& Ria, L. (2007). Devenir enseignant. Parcours et formation. Paris : Armand Colin.

Guibert, P., Lazuech, G., \& Rimbert, F. (2008). Enseignants débutants, " faire ses classes ». L'insertion professionnelle des professeurs du second degré. Rennes: Presses universitaires de Rennes.

Lahire, B. (1998). L'homme pluriel. Les ressorts de l'action. Paris : Nathan.

Lave, J., \& Wenger, E. (1991). Situated learning : legitimate peripheral participation. New York : Cambridge University Press.

Leblanc, S., Ria, L., Dieumegard, G., Serres, G., \& Durand, M. (2008). Concevoir des dispositifs de formation professionnelle des enseignants à partir de l'analyse de l'activité dans une approche enactive. @ctivités, 5(1), 58-78.

Livret vert (2008). http://media.education.gouv.fr/file/Commission_Pochard.

Maroy C. (2006). Les évolutions du travail enseignant en France et en Europe : facteurs de changement, incidences et résistances dans l'enseignement secondaire. Revue Française de Pédagogie, 155, 112-142.

Pastré, P. (1995). Problèmes didactiques posés par les simulations. Performances Humaines et Techniques, 75/76, 44-53.

Pastré, P. (2008). La didactique professionnelle : origines, fondements, perspectives. Travail et Apprentissage, 1, 9-21.

Rayou, P., \& van Zanten, A. (2004). Enquête sur les nouveaux enseignants. Changeront-ils l'école? Paris : Editions Bayard.

Ria, L., Leblanc, S., Serres, G., \& Durand, M. (2006). Recherche et formation en « analyse des pratiques » : un exemple d'articulation. Recherche et Formation, 51, 43-56.

Ria, L., \& Rayou, P. (2008). Sociologie et ergonomie cognitive au miroir des situations éducatives: le cas de l'entrée dans le métier des enseignants du second degré. Recherches \& Educations, 1, 105-119.

Ria, L., Saury, J., Sève, C., \& Durand, M. (2001). Les dilemmes des enseignants débutants : études lors des premières expériences de classe en Education Physique. Science et Motricité, 42, 47-58. 
Ria, L., Sève, C., Theureau, J., Saury, J., \& Durand, M. (2003). Beginning teacher's situated emotions: Study about first classroom's experiences. Journal of Education for Teaching, 29(3), 219-233.

Ria, L., Sève, C., Durand, M., \& Bertone, B. (2004). Indétermination, contradiction et exploration : trois expériences typiques des enseignants débutants en Education Physique. Revue des Sciences de l'Education, XXX (3), 535-554.

Rosch, E. (1978). Principles of categorization. In E. Rosch, \& B.B. Llyod (Eds.), Cognition and categorization (pp.27-48). Hillsdale, NJ : Lauwrence Erlbaum Associates.

Rouve, M., \& Ria, L. (2008). Analyse de l'activité professionnelle d'enseignants néotitulaires en réseau ambition réussite : études de cas. Travail \& Formation en Education. Revue en ligne : http://tfe.revues.org/

Serres, G., Ria, L., \& Adé, D. (2004). Modalités de développement de l'activité professionnelle au gré des contextes de classe et de formation : le cas des professeurs stagiaires en Education Physique et Sportive. Revue Française de Pédagogie, 149, 4964.

Sgen-CFDT (2008). Profession Éducation. Une enquête rigoureuse et anonyme sur toutes les dimensions des métiers. Supplément au numéro 175. www.sgen-cfdt.org.

Smethem, L. (2007). Retention and intention in teaching careers: Will the new generation stay? Teachers and Teaching: Theory and Practice, 13(5), 465-480.

Theureau, J. (2004). Le cours d'action : Méthode élémentaire. Toulouse : Octarès.

Theureau, J. (2006). Le cours d'action : Méthode développée. Toulouse : Octarès.

Varela, F.J. (1989). Autonomie et connaissance. Essai sur le vivant. Paris : Seuil. 